\title{
Charge Density and Hydrophobicity-Dominated Regimes in the Phase Behavior of Complex Coacervates
}

\author{
Jun Huang and Jennifer E. Laaser* \\ Department of Chemistry, University of Pittsburgh, Pittsburgh, PA 15260 \\ E-mail: j.laaser@pitt.edu
}

Phone: (412)383-0125

\begin{abstract}
The role of hydrophobicity, and particularly nonionic hydrophobic comonomers, on the phase behavior of polyelectrolyte complex coacervates is not well-understood. Here, we address this problem by synthesizing a library of polymers with a wide range of charge densities and nonionic hydrophobic side chain lengths, and characterizing their phase behavior by optical turbidity. The polymers were prepared by postpolymerization modification of poly(N-acryloxy succinimide), targeting charge densities between 40 and $100 \%$ and nonionic aliphatic sidechains with lengths from 0 to 12 carbons long. Turbidity measurements on pairs of polycations and polyanions with matched charge densities and nonionic sidechain lengths revealed a complex salt response with distinct charge density-dominated and hydrophobicity-dominated regimes. The polymer solubilities were not directly correlated with the phase behavior of the coacervates, indicating the difficulty of understanding the coacervate phase behavior in terms of the polymer-water interaction parameter. This result suggests that there
\end{abstract}


is significant room for further work to understand the mechanisms by which specific molecular-scale interactions moderate the phase behavior of complex coacervates.

Complex coacervates are a class of materials prepared by mixing aqueous solutions of two oppositely charged polyelectrolytes. Associative phase separation between the polymers leads to the formation of a dense, polymer-rich coacervate phase and a dilute, polymer-poor supernatant phase. ${ }^{1}$ The charged species involved in the process can include colloids, proteins, surfactants, or polymers. ${ }^{2}$ The resulting materials have a wide range of applications in fields such as nucleic acid delivery, protein encapsulation, adhesives, and salt-processable materials. ${ }^{3-8}$ The physical properties of these materials, such as their phase behavior and viscoelastic properties, are important for tailoring polyelectrolyte complexes for such applications. ${ }^{1}$ Therefore, systematically understanding the material properties of polyelectrolyte complex coacervates, and how they depend on the chemistry and physics of the polyelectrolytes, is the key to future functional material design.

Many parameters, such as solution conditions, polymer architecture, and composition can influence the phase behavior and viscoelastic properties of polyelectrolyte complex coacervates. $^{9-12}$ To date, most research has focused on the role of solution conditions like $\mathrm{pH}$, temperature, and ionic strength. ${ }^{13-15}$ In addition to the external solution conditions, however, chemical factors such as the polymer composition should also have a significant impact on both the phase behavior and bulk material properties of complex coacervates, ${ }^{16-18}$ and may offer a viable route to tuning these properties when the solution conditions are fixed. Recent theoretical and experimental studies have shown, for example, that decreasing the charge density of the polyelectrolytes reduces the salt resistance of the complexes and speeds up their relaxation dynamics. ${ }^{17,19}$ Hydrophobicity also appears to play an important role: increasing the hydrophobicity of the charged sidechain, either by adding additional hydrophobic chains directly to the charged unit or by changing the polarity of the linker connecting it to the polymer backbone, or increasing the hydrophobicity of the polymer backbone, typically increases the salt resistance of the resulting complexes. ${ }^{20-22}$ 
Interestingly, however, the effect of hydrophobic groups appears to depend on exactly how they are incorporated into the polymers. When all of the repeat units of the polymer are made more hydrophobic, either by modifying the backbone or the charged sidechains, the salt resistance of the coacervates typically increases. ${ }^{22,23}$ When hydrophobic groups are incorporated as nonionic comonomers, however, different trends have been observed. In short polypeptide systems, for example, switching the nonionic, hydrophobic residues from glycine, to alanine, and to leucine induces measurable shifts in the apparent salt resistance of the complexes, on the order of $15-50 \mathrm{mM}$ depending on the peptides' charge densities. ${ }^{24}$ In a recent study from our group, on the other hand, adding 4-carbon butyl sidechains to the nonionic repeat units in acrylamide-based polyelectrolytes appeared to have no effect on either the phase behavior or the rheology of the resulting coacervates. ${ }^{25}$ While these studies are apparently contradictory, the fact that they were carried out on different polymer systems with different chain lengths and different ranges of charge densities makes it difficult to develop a comprehensive picture of how nonionic, hydrophobic sidechains influence coacervate behavior.

Here, we address this problem by investigating the phase behavior of coacervates formed from a library of 54 acrylamide-based copolymers with charge densities ranging from 40-100\% and nonionic aliphatic sidechains ranging from zero to twelve carbons in length. This range of compositions spans a wide range of charge densities and hydrophobicities, and allows systematic investigation of the role of hydrophobic nonionic sidechains in complex coacervates. The phase behavior of the resulting coacervates was characterized by optical turbidity, revealing three distinct regimes of complex coacervate behavior. For polymers with hydrophilic sidechains, the salt resistance of the polymers decreases with decreasing charge density, while for polymers with the longest hydrophobic sidechains, the salt resistance increases with increasing hydrophobic content, as expected from simple models for complex coacervation. ${ }^{16,26}$ For polymers with intermediate length hydrophobic sidechains (C2-C4), however, there is a critical charge density at which the phase behavior switches from being dominated by the 
charge density to being dominated by the hydrophobic interactions. Comparison with the solubilities of the component polyelectrolytes reveals that the phase behavior is not simply determined by changes in the polymer solubility, suggesting that understanding the role of hydrophobic interactions in coacervates of polyelectrolyte copolymers will require a detailed understanding of the molecular interactions involved in these systems.

a)
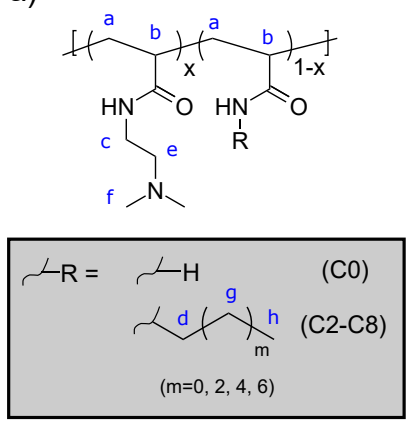

b)

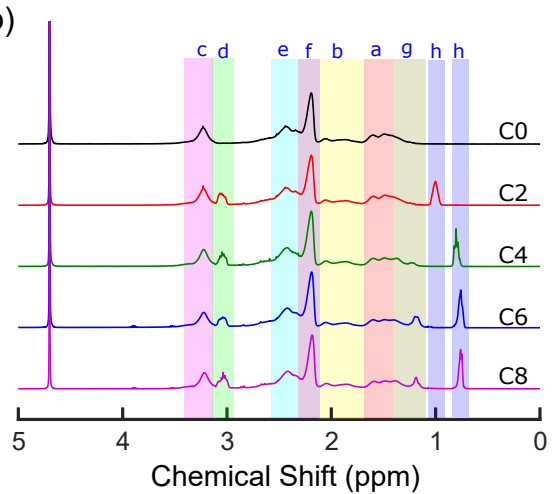

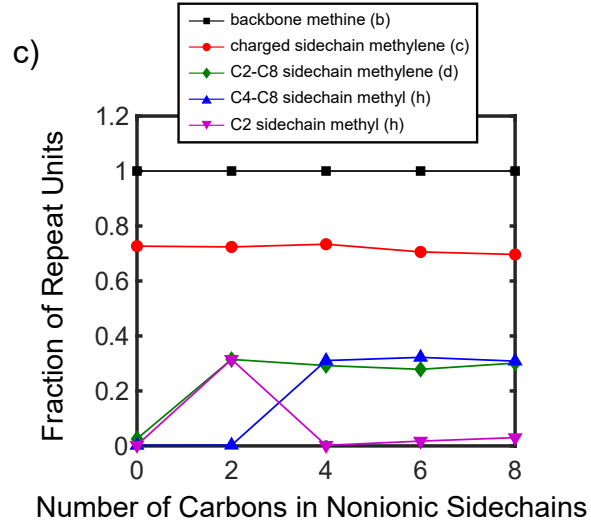

Figure 1: (a) Structure and (b) representative NMR spectra of tertiary amine-bearing polyelectrolytes with constant charge density (70\%) and varying hydrophobic sidechain length, with (c) normalized integrals of key peaks illustrating the consistency of the charge densities achieved using the batch-splitting method described in the text.

The polymer library was synthesized by post-polymerization functionalization of poly $(\mathrm{N}-$ acryloxy succinimide) (poly(NAS)). ${ }^{25}$ Using this approach, we prepared a library of 54 polyelectrolytes with acidic (carboxylic acid) or basic (tertiary amine) side chains on a subset of the monomers, and hydrophilic (amide) or hydrophobic (ethyl, butyl, hexyl, octyl, decyl, and dodecyl) side chains on the remaining monomers. Preparation by post-polymerization functionalization ensures that all of the synthesized polyelectrolytes have the same degree of polymerization and dispersity, eliminating chain length differences as a potential source of observed differences in the phase behavior. The synthetic approach was additionally designed to ensure that all polymers with the same charge and charge density did, indeed, have exactly the same fraction of charged units, even when the hydrophobic amines have different reactivities. To this end, a large batch of the poly(NAS) was first substituted with the targeted fraction of charged comonomers. The reaction mixture was then divided into 
several fractions, and a different nonionic amine compound was added to each fraction. The polyelectrolyte compositions were quantified by NMR, which confirmed that the described synthetic approach did indeed produce polymers with effectively identical charge densities (Fig. 1 and Supporting Information).

Table 1: Summary of polymer compositions investigated in this work

\begin{tabular}{ccccccc}
\hline \multirow{2}{*}{ Nonionic Sidechain } & \multicolumn{6}{c}{ Charge Density (\%) } \\
& 90 & 80 & 70 & 60 & 50 & 40 \\
\hline C0 & $\checkmark$ & $\checkmark$ & $\checkmark$ & $\checkmark$ & $\checkmark$ & $\checkmark$ \\
C2 & $\checkmark$ & $\checkmark$ & $\checkmark$ & $\checkmark$ & $\checkmark$ & $\checkmark$ \\
C4 & $\checkmark$ & $\checkmark$ & $\checkmark$ & $\checkmark$ & $\checkmark$ & \\
C6 & $\checkmark$ & $\checkmark$ & $\checkmark$ & $\checkmark$ & & \\
C8 & $\checkmark$ & $\checkmark$ & $\checkmark$ & & & \\
C10 & $\checkmark$ & $\checkmark$ & & & & \\
C12 & $\checkmark$ & & & & & \\
\hline
\end{tabular}

The compositions of the resulting polymers are summarized in Table 1. As shown in this table, not all sidechain lengths were prepared at all charge densities; the long hydrophobic sidechains were omitted from the polymer library at low charge densities on the basis of preliminary experiments which indicated that these polymers had too low of a solubility to be useful in turbidity experiments.

The phase behavior of the complexes formed from oppositely-charged polyelectrolytes with identical charge densities and hydrophobic sidechains was then characterized by optical turbidity. In this experiment, coacervates were formed via mixing stock solutions of oppositely charged polymers with $\mathrm{KCl}$ to reach each targeted composition, and the apparent absorbance of each sample was characterized using a UV-vis spectrometer. To maintain consistent charge on the weak acidic and basic sidechains across experiments, all samples were prepared in a standard buffer (bis-tris, $0.1 \mathrm{M}$ ionic strength, $\mathrm{pH}=6.5$ ). As seen in Figure 2, the apparent absorbance was high at low salt concentration, indicating that the samples phase separated into droplets that scattered light away from the detector. As the salt concentration increased, the absorbance decreased until it reached a baseline indistinguishable from the measured absorbance of pure water. This limit reflects formation of a single-phase 

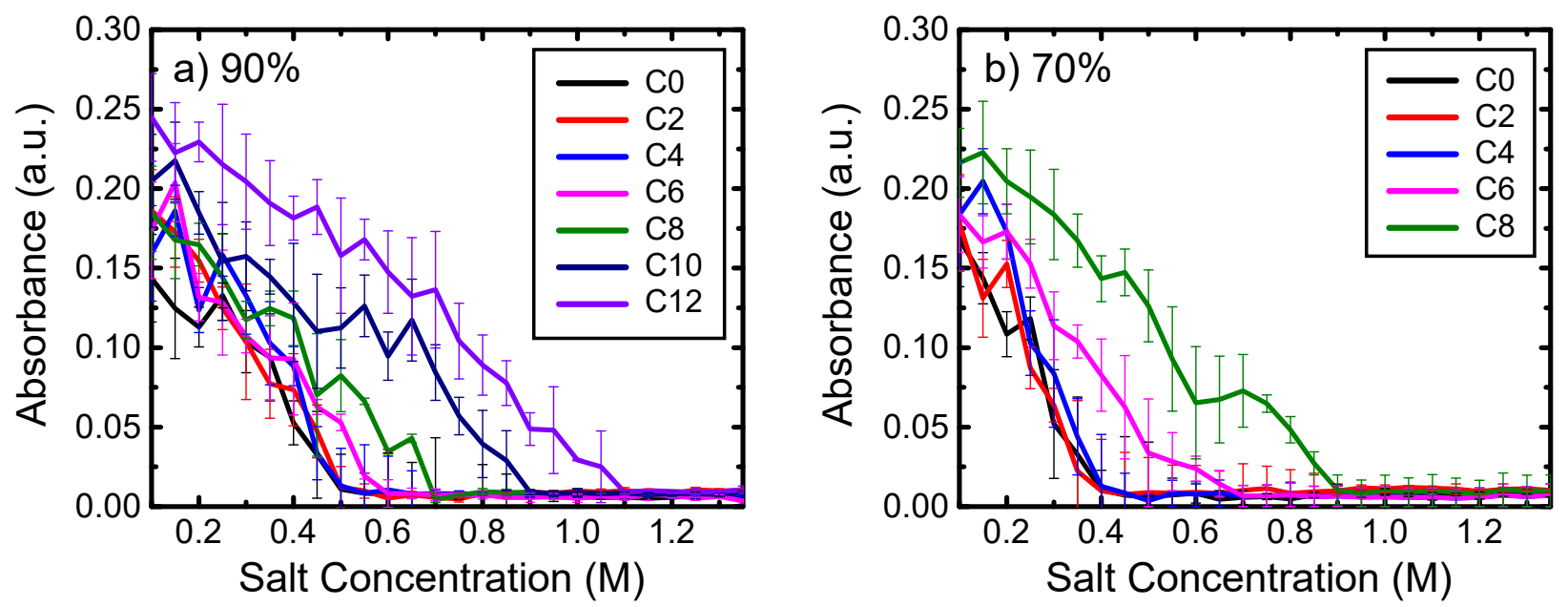

Figure 2: Representative turbidities of coacervate samples prepared using polyelectrolytes with (a) 90\% charged monomers and (b) $70 \%$ charged monomers at a total charged monomer concentration of $0.001 \mathrm{M}$ and varying salt concentrations. Vertical error bars represent the standard deviation of three measurements. Full turbidity data for all polymer pairs is included in the Supporting Information.

polymer solution, where no phase separation occurs and little light is scattered from the sample. ${ }^{27}$ As seen in Figure 2, for coacervates with the same charge density, the salt concentration at which the system transitioned from two phases (high absorbance) to one phase (low absorbance), referred to as the salt resistance, ${ }^{28}$ generally increased for systems with longer hydrophobic nonionic sidechains in their component polyelectrolytes. Interestingly, for polymers with short sidechains $(\mathrm{C} 0-\mathrm{C} 4)$, this transition occurred at a lower salt concentration in the polymers with lower charge densities, while for the polymers with longer C8 sidechains, the transition occurred at a higher salt concentration in the polymers with the lower charge densities.

To quantify this behavior, the salt resistance was determined as the point at which the turbidity first dropped to within three standard deviations of the high-salt-concentration baseline. ${ }^{25}$ The resulting salt resistance for all of the coacervate systems investigated in this work is summarized in Figure 3. As seen in this figure, the salt resistance of samples with hydrophilic (amide) nonionic comonomers decreased with decreasing charge density, as expected, ${ }^{16}$ while for coacervate samples with larger hydrophobic (octyl, decyl, dodecyl) 


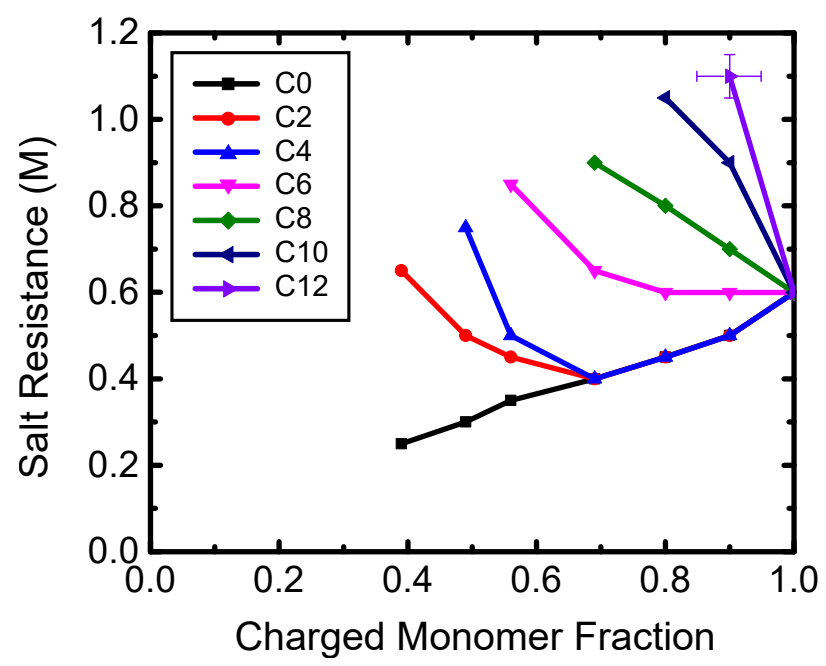

Figure 3: Salt resistance of coacervates prepared at a total charged monomer concentration of $0.001 \mathrm{M}$ as a function of charge density and sidechain length. Representative error bar indicates estimated $\pm 5 \mathrm{~mol} \%$ uncertainty in the compositions determined by NMR, and $\pm 0.05 \mathrm{M}$ uncertainty in the salt resistances based on the step size used in the turbidity measurements. ${ }^{25}$

nonionic comonomer side chains, the salt resistance increased with decreasing charge density. For samples with shorter hydrophobic nonionic comonomers (C0, C2, and $\mathrm{C} 4$ sidechains), the dependence of the salt resistance on charge density is nonmonotonic, and there exists a turnover charge density. Above the turnover point, the salt resistance decreased with decreasing charge density, while below the charge density, the salt resistance increased with decreasing charge density. While this behavior at first glance appears to contradict simple models of complex coacervation which predict that the critical salt concentration (for which salt resistance is a useful proxy) should always decrease with decreasing charge density because of the decreased entropy gain upon counterion release, ${ }^{16}$ we note that this prediction only strictly holds if the interaction parameter $(\chi)$ for polymer-water interactions remains constant. Because the total hydrophobic content of the polymers increases as the charge density decreases, however, the interaction parameter should increase as the charge density decreases. ${ }^{23}$ As a result, the magnitude of this effect should be strongest for the longest alkyl chains, in which the marginal increase in hydrophobic content for each charged monomer that is replaced by a hydrophobic monomer is greatest. Interestingly, however, for polymers 
at the same charge density, the salt resistance is almost identical for polymers with short alkyl chains ( $\mathrm{C} 0-\mathrm{C} 4$ at $70 \%$ charge and above), even though these polymers should have increasingly unfavorable interactions with the surrounding water that should systematically increase the critical salt concentration.
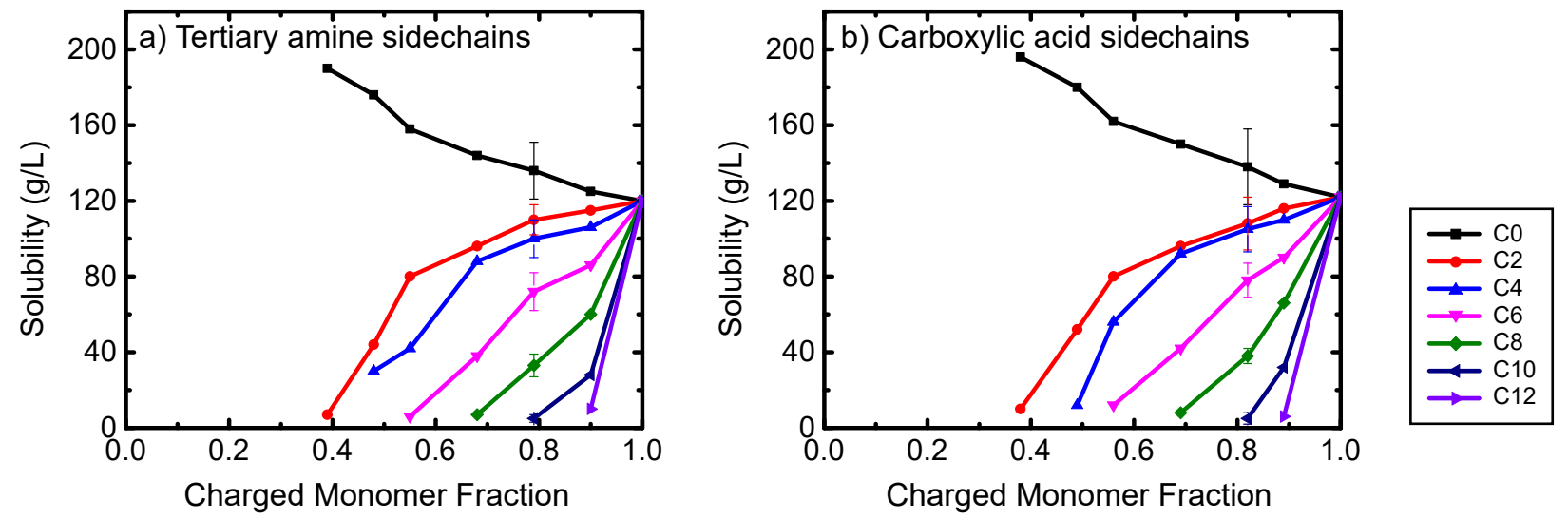

Figure 4: Solubility of (a) positively charged and (b) negatively charged polyelectrolytes as a function of charge density and sidechain length. Representative error bars show the typical reproducibility achieved in three independent solubility measurements.

Because increasing the $\chi$ parameter for the polymer-water interaction should also decrease the solubility of the polymers, we hypothesized that the salt resistance of the coacervates and the solubility of the polyelectrolytes should be strongly correlated. To test this hypothesis, we measured the solubility of all 54 polymers in the same buffer used in the turbidity experiments. The resulting solubilities are shown in Figure 4, and their correlation with the salt resistances is shown in Figure 5. As seen in Figure 4, the solubility of the polymers with hydrophilic amide sidechains increased as the charge density decreased, while the solubilities of all polyelectrolytes with hydrophobic nonionic sidechains decreased as the charge density decreased, with steeper decreases observed for polymers with longer alkyl chains. The solubility trends were similar for positively- and negatively-charged polymers, with only small difference in the solubility values for polymers with the same charge density and nonionic sidechain.

The relationship between salt resistance and solubility, shown in Figure 5, exhibits a number of interesting features. First, the data bifurcates around the point for the $100 \%$ 


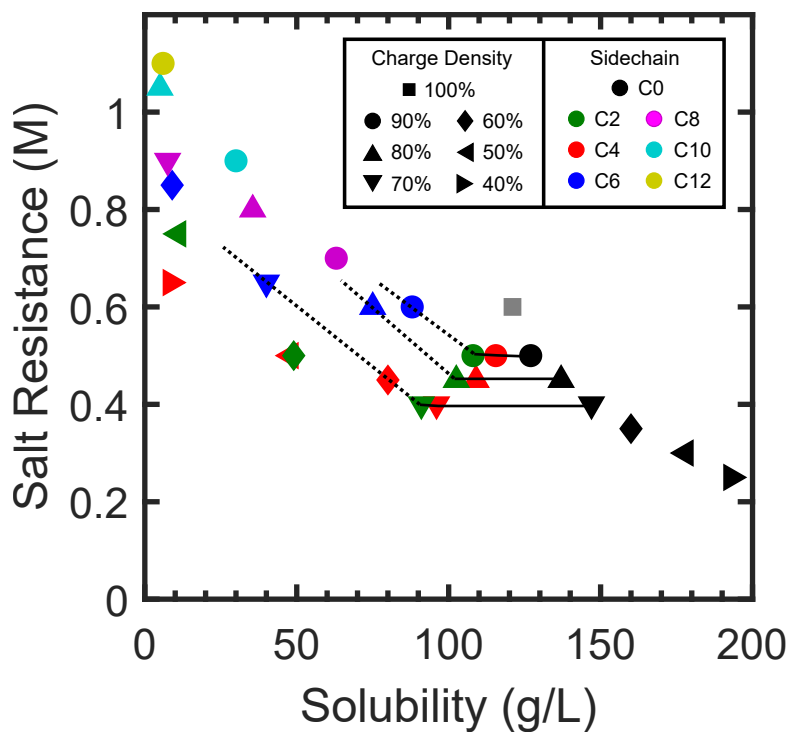

Figure 5: Salt resistance of polyelectrolyte complex coacervates with varying charge densities and sidechain lengths as a function of the average solubility of the constituent polyelectrolytes. Lines connect representative points within the same charge density series, illustrating the independence of salt resistance on solubility discussed in the text.

charged polymers. The systems with hydrophobic alkyl-chain comonomers fall to the left of this point, with lower solubilities, while the systems with hydrophilic amide comonomers fall to the right, with higher solubilities. For the polymers with amide $(\mathrm{C} 0)$ comonomers, the salt resistance decreases monotonically as the solubility increases. For these polyelectrolytes, the decrease in salt resistance could arise solely from the decrease in charge density and the resulting decrease in the entropy gain from counterion release upon coacervate formation. ${ }^{29}$ However, the increase in solubility also suggests a more favorable polymer-water interaction parameter as additional hydrophilic monomers are introduced. ${ }^{30}$ For the polymers with alkyl sidechains (C2-C12), on the other hand, there appear to be two regimes: in systems with short hydrophobic sidechains $(\mathrm{C} 2, \mathrm{C} 4)$, the salt resistance decreases and then increases with decreasing solubility, while for systems with longer hydrophobic sidechains, the salt resistance increases with decreasing solubility. Within each series with the same charge density, we also find that the polymers with short $(\mathrm{C} 0-\mathrm{C} 2)$ sidechains typically have very similar salt resistances even when their solubilities vary by up to $50 \%$, although this trend disappears at 
charge densities of $60 \%$ and below. These trends clearly show that the salt resistance is not well predicted by the polymer solubility, even for polymers with the same charge density. This result suggests that the role of hydrophobicity in shaping the phase behavior of polymer based complex coacervates cannot be explained as a result of changes in the polymer-water interaction parameter alone. For the polymers prepared at $70 \%$ charge density, for example, the $\mathrm{C} 0, \mathrm{C} 2$, and $\mathrm{C} 4$ systems have solubilities that differ by almost $50 \%$, indicating that they have significantly different polymer-water interaction parameters. Since their charge densities are the same, current models of complex coacervation would predict that their critical salt concentrations and salt resistances should also differ, with the system having the highest interaction parameter (the polymers with $\mathrm{C} 4$ sidechains) predicted to have the highest salt resistance. ${ }^{16}$ However, the data show that these polymers in fact have nearly identical salt resistances, despite their different solubilities. We thus conclude that the effect of hydrophobic groups on the phase behavior of complex coacervates cannot be understood solely in terms of an increase in the polymer-water interaction parameter, and that more complex mechanisms must be considered.

One possible origin for this discrepancy is that the interaction parameter in models of complex coacervation is typically adapted from Flory-Huggins theory, which is itself an extension of regular solution theory, where the interaction parameter reflects the enthalpy of solute-solvent interactions. ${ }^{31}$ On a macroscopic scale, the immiscibility of hydrophobic materials with water is indeed often thought of as an enthalpic effect, in which creating water-oil interfaces requires unfavorable interactions that are energetically costly. ${ }^{32}$ However, at the molecular scale, and particularly when considering the hydration of polymers at the monomer or sidechain level, hydrophobicity has a significant entropic component. ${ }^{33}$ Free energies of hydration for polymers with aromatic sidechains measured by single-molecule force experiments, for example, show a maximum with increasing temperature that is characteristic of the entropic penalty for creating a cavity in the water in which to contain the hydrophobic chains. ${ }^{34}$ Interestingly, in that work the critical length scale at which the hydra- 
tion behavior appears to transition from microscopic (entropic) to macroscopic (enthalpic) is approximately $1 \mathrm{~nm} ;{ }^{34}$ this correlates well with the extended chain length of the C8 chains above which we see hydrophobic behavior strongly dominate both the solubilities and salt resistances in the present experiments.

In this context, the complicated effects of hydrophobicity and charge density in shaping the phase behavior of complex coacervates may reflect a tradeoff between the entropic gains from counterion release upon ion pairing (which decreases in magnitude as the charge density decreases) and from water release upon hydrophobic aggregation (which increases in magnitude as the total hydrophobic content of the polymer increases). For polyelectrolytes with high charge densities and less hydrophobic sidechains, counterion release dominates the phase behavior of the coacervates. ${ }^{29}$ For polyelectrolytes with larger hydrophobic side chains, on the other hand, the contribution from hydrophobicity plays a dominant role. ${ }^{33}$ This hypothesis, and the complicated tradeoff between charge density and hydrophobic content observed in the present system, thus suggests several interesting directions for future work. First and foremost, careful thermodynamic measurements should help clarify the role of entropic vs. enthalpic contributions in the coacervation of hydrophobic polyelectrolytes. Quantification of the full phase diagram, and particularly the partitioning of salt between the coacervate and supernatant phases, may provide insight into whether changing the hydrophobic sidechain lengths further affects the phase behavior by changing the effective dielectric constant of each phase. ${ }^{23}$ Scattering measurements may additionally provide insight into whether the hydrophobic content drives a difference in the chain collapse and/or compaction of the polymer chains before or after coacervation. And experiments in which the molecular weight and backbone chemistry of the polymers are varied may help reveal why other polymer systems appear to be more sensitive to small changes in the hydrophobic sidechain size than the polyacrylamides investigated in this work.

In summary, we have shown that the role of nonionic, hydrophobic sidechains in determining the phase behavior of complex coacervates is more complex than predicted by current 
theories of complex coacervation. The synthetic approach used in the present work allows us to prepare polyelectrolyte libraries with a wide range of hydrophobic sidechains while maintaining identical charge densities and molecular weight distributions. Any changes in the phase behavior must result thus from changes in the hydrophobic interactions between the nonionic sidechains. As shown in our data, the salt resistance of the resulting coacervates is not well-predicted by the solubility of the constituent polymers. Based on this result, we conclude that hydrophobicity in complex coacervates cannot be accounted for using changes in the enthalpic interaction parameter alone, and both careful thermodynamic characterization and updated models for coacervate phase behavior will need to be developed that more accurately understand and account for the entropic contributions to hydrophobic hydration.

\section{Acknowledgement}

The authors thank the donors of the American Chemical Society Petroleum Research Fund for support of this research (Award No. 58034-DNI7)

\section{Supporting Information Available}

Experimental procedures, characterization data (NMR, SEC, and solubility) for synthesized polymers, and full turbidity data for all polymer pairs reported in this work.

\section{References}

(1) Sing, C. E.; Perry, S. L. Recent progress in the science of complex coacervation. Soft Matter 2020, 16, 2885-2914.

(2) Zhou, L.; Shi, H.; Li, Z.; He, C. Recent Advances in Complex Coacervation Design from Macromolecular Assemblies and Emerging Applications. Macromolecular Rapid Communications 2020, 41, 2000149. 
(3) Samal, S. K.; Dash, M.; Vlierberghe, S. V.; Kaplan, D. L.; Chiellini, E.; van Blitterswijk, C.; Moroni, L.; Dubruel, P. Cationic polymers and their therapeutic potential. Chemical Society Reviews 2012, 41, 7147.

(4) Black, K. A.; Priftis, D.; Perry, S. L.; Yip, J.; Byun, W. Y.; Tirrell, M. Protein Encapsulation via Polypeptide Complex Coacervation. ACS Macro Letters 2014, 3, 1088-1091.

(5) Wei, W.; Tan, Y.; Rodriguez, N. R. M.; Yu, J.; Israelachvili, J. N.; Waite, J. H. A mussel-derived one component adhesive coacervate. Acta Biomaterialia 2014, 10, 1663-1670.

(6) Schaaf, P.; Schlenoff, J. B. Saloplastics: Processing Compact Polyelectrolyte Complexes. Advanced Materials 2015, 27, 2420-2432.

(7) Meng, X.; Perry, S. L.; Schiffman, J. D. Complex Coacervation: Chemically Stable Fibers Electrospun from Aqueous Polyelectrolyte Solutions. ACS Macro Letters $\mathbf{2 0 1 7}$ $6,505-511$.

(8) Blocher, W. C.; Perry, S. L. Complex coacervate-based materials for biomedicine. Wiley Interdisciplinary Reviews: Nanomedicine and Nanobiotechnology 2016, 9, e1442.

(9) Dompé, M.; Cedano-Serrano, F. J.; Vahdati, M.; Sidoli, U.; Heckert, O.; Synytska, A.; Hourdet, D.; Creton, C.; van der Gucht, J.; Kodger, T.; Kamperman, M. Tuning the Interactions in Multiresponsive Complex Coacervate-Based Underwater Adhesives. International Journal of Molecular Sciences 2019, 21, 100.

(10) Lytle, T. K.; Sing, C. E. Tuning chain interaction entropy in complex coacervation using polymer stiffness, architecture, and salt valency. Molecular Systems Design E Engineering 2018, 3, 183-196.

(11) Wang, Q.; Schlenoff, J. B. The Polyelectrolyte Complex/Coacervate Continuum. Macromolecules 2014, 47, 3108-3116. 
(12) Priftis, D.; Tirrell, M. Phase behaviour and complex coacervation of aqueous polypeptide solutions. Soft Matter 2012, 8, 9396-9405.

(13) Shamoun, R. F.; Hariri, H. H.; Ghostine, R. A.; Schlenoff, J. B. Thermal Transformations in Extruded Saloplastic Polyelectrolyte Complexes. Macromolecules 2012, 45, 9759-9767.

(14) Liu, Y.; Momani, B.; Winter, H. H.; Perry, S. L. Rheological characterization of liquidto-solid transitions in bulk polyelectrolyte complexes. Soft Matter 2017, 13, 7332-7340.

(15) Jha, P.; Desai, P.; Li, J.; Larson, R. pH and Salt Effects on the Associative Phase Separation of Oppositely Charged Polyelectrolytes. Polymers 2014, 6, 1414-1436.

(16) Spruijt, E.; Westphal, A. H.; Borst, J. W.; Stuart, M. A. C.; van der Gucht, J. Binodal Compositions of Polyelectrolyte Complexes. Macromolecules 2010, 43, 6476-6484.

(17) Radhakrishna, M.; Basu, K.; Liu, Y.; Shamsi, R.; Perry, S. L.; Sing, C. E. Molecular Connectivity and Correlation Effects on Polymer Coacervation. Macromolecules 2017, 50, 3030-3037.

(18) Chang, L.-W.; Lytle, T. K.; Radhakrishna, M.; Madinya, J. J.; Vélez, J.; Sing, C. E.; Perry, S. L. Sequence and entropy-based control of complex coacervates. Nature Communications 2017, 8 .

(19) Ali, S.; Prabhu, V. Relaxation Behavior by Time-Salt and Time-Temperature Superpositions of Polyelectrolyte Complexes from Coacervate to Precipitate. Gels 2018, 4, 11.

(20) Zhang, Z.; Chen, Q.; Colby, R. H. Dynamics of associative polymers. Soft Matter 2018, 14, 2961-2977. 
(21) Lou, J.; Friedowitz, S.; Qin, J.; Xia, Y. Tunable Coacervation of Well-Defined Homologous Polyanions and Polycations by Local Polarity. ACS Central Science 2019, 5, $549-557$.

(22) Sadman, K.; Wang, Q.; Chen, Y.; Keshavarz, B.; Jiang, Z.; Shull, K. R. Influence of Hydrophobicity on Polyelectrolyte Complexation. Macromolecules 2017, 50, 94179426.

(23) Liu, Y.; Chalarca, C. F. S.; Carmean, R. N.; Olson, R. A.; Madinya, J.; Sumerlin, B. S.; Sing, C. E.; Emrick, T.; Perry, S. L. Effect of Polymer Chemistry on the Linear Viscoelasticity of Complex Coacervates. Macromolecules 2020, 53, 7851-7864.

(24) Tabandeh, S.; Leon, L. Engineering Peptide-Based Polyelectrolyte Complexes with Increased Hydrophobicity. Molecules 2019, 24, 868.

(25) Huang, J.; Morin, F. J.; Laaser, J. E. Charge-Density-Dominated Phase Behavior and Viscoelasticity of Polyelectrolyte Complex Coacervates. Macromolecules 2019, 52, $4957-4967$.

(26) Spruijt, E.; Stuart, M. A. C.; van der Gucht, J. Linear Viscoelasticity of Polyelectrolyte Complex Coacervates. Macromolecules 2013, 46, 1633-1641.

(27) Perry, S.; Li, Y.; Priftis, D.; Leon, L.; Tirrell, M. The Effect of Salt on the Complex Coacervation of Vinyl Polyelectrolytes. Polymers 2014, 6, 1756-1772.

(28) Madinya, J. J.; Chang, L.-W.; Perry, S. L.; Sing, C. E. Sequence-dependent selfcoacervation in high charge-density polyampholytes. Molecular Systems Design $\&$ Engineering 2020, 5, 632-644.

(29) Fu, J.; Schlenoff, J. B. Driving Forces for Oppositely Charged Polyion Association in Aqueous Solutions: Enthalpic, Entropic, but Not Electrostatic. Journal of the American Chemical Society 2016, 138, 980-990. 
(30) Mikos, A. G.; Peppas, N. A. Flory interaction parameter X for hydrophilic copolymers with water. Biomaterials 1988, 9, 419-423.

(31) Russell, T. H.; Edwards, B. J.; Khomami, B. Characterization of the Flory-Huggins interaction parameter of polymer thermodynamics. EPL (Europhysics Letters) 2014, $108,66003$.

(32) Li, I. T. S.; Walker, G. C. Interfacial Free Energy Governs Single Polystyrene Chain Collapse in Water and Aqueous Solutions. Journal of the American Chemical Society 2010, 132, 6530-6540.

(33) Li, I. T. S.; Walker, G. C. Single Polymer Studies of Hydrophobic Hydration. Accounts of Chemical Research 2012, 45, 2011-2021.

(34) Li, I. T. S.; Walker, G. C. Signature of hydrophobic hydration in a single polymer. Proceedings of the National Academy of Sciences 2011, 108, 16527-16532. 